\title{
No Evidence for Axions from Chandra Observation of the Magnetic White Dwarf RE J0317-853
}

\author{
Christopher Dessert $\odot,{ }^{1,2,3}$ Andrew J. Long $\odot,{ }^{4}$ and Benjamin R. Safdi $\oplus^{2,3}$ \\ ${ }^{1}$ Leinweber Center for Theoretical Physics, Department of Physics, University of Michigan, Ann Arbor, Michigan 48109, USA \\ ${ }^{2}$ Berkeley Center for Theoretical Physics, University of California, Berkeley, California 94720, USA \\ ${ }^{3}$ Theoretical Physics Group, Lawrence Berkeley National Laboratory, Berkeley, California 94720, USA \\ ${ }^{4}$ Department of Physics and Astronomy, Rice University, Houston, Texas 77005, USA
}

(Received 3 May 2021; revised 7 September 2021; accepted 19 January 2022; published 18 February 2022)

Axions with couplings $g_{\text {ary }} \sim$ few $\times 10^{-11} \mathrm{GeV}^{-1}$ to electromagnetism may resolve a number of astrophysical anomalies, such as unexpected $\sim \mathrm{TeV}$ transparency, anomalous stellar cooling, and $\mathrm{x}$-ray excesses from nearby neutron stars. We show, however, that such axions are severely constrained by the nonobservation of $\mathrm{x}$ rays from the magnetic white dwarf (MWD) RE J0317-853 using 40 ks of data acquired from a dedicated observation with the Chandra X-ray Observatory. Axions may be produced in the core of the MWD through electron bremsstrahlung and then convert to $\mathrm{x}$ rays in the magnetosphere. The nonobservation of $\mathrm{x}$ rays constrains the axion-photon coupling to $g_{a \gamma \gamma} \lesssim 5.5 \times 10^{-13} \sqrt{C_{a \gamma \gamma} / C_{a e e}} \mathrm{GeV}^{-1}$ at 95\% confidence for axion masses $m_{a} \lesssim 5 \times 10^{-6} \mathrm{eV}$, with $C_{a e e}$ and $C_{a \gamma \gamma}$ the dimensionless coupling constants to electrons and photons. Considering that $C_{a e e}$ is generated from the renormalization group, our results robustly disfavor $g_{a \gamma \gamma} \gtrsim 4.4 \times 10^{-11} \mathrm{GeV}^{-1}$ even for models with no ultraviolet contribution to $C_{a e e}$.

DOI: 10.1103/PhysRevLett.128.071102

Axions are hypothetical pseudoscalar particles that couple through dimension-5 operators to the standard model. In particular the quantum chromodynamics (QCD) axion couples to QCD, which allows it to solve the strong- $C P$ problem [1-4]; this coupling also generates a mass $m_{a}^{\mathrm{QCD}} \sim \Lambda_{\mathrm{QCD}}^{2} / f_{a}$ for the particle, with $f_{a}$ the axion decay constant and $\Lambda_{\mathrm{QCD}}$ the QCD confinement scale. In this Letter we probe axions with masses $m_{a} \lesssim 10^{-2} \mathrm{eV}$ that do not necessarily couple to QCD (but see Refs. [5-8]) though they couple to electromagnetism and matter. Such axions, often referred to as axionlike particles, are especially motivated theoretically in the context of the String axiverse [9-14]. In the axiverse it is natural to expect a large number $N$ of light axions, with $m_{a} \ll m_{a}^{\mathrm{QCD}}$. One linear combination couples to QCD and receives a mass from QCD, becoming the QCD axion, while the rest of the $N-1$ states remain light and retain their non-QCD couplings to the standard model. It is well established that axions may be produced within stars including white dwarfs (WDs) (see, e.g., Refs. [15-17]) and escape the stars due to their weak interaction strengths with matter. Recently we pointed out that such axions could produce $\mathrm{x}$-ray signatures through

Published by the American Physical Society under the terms of the Creative Commons Attribution 4.0 International license. Further distribution of this work must maintain attribution to the author(s) and the published article's title, journal citation, and DOI. Funded by SCOAP ${ }^{3}$. axion-photon conversion in magnetic WD (MWD) magnetospheres in our previous paper [18] [see Refs. [19-28] for related discussions about axion-photon conversion in neutron star (NS) magnetospheres]. In this Letter we collect and analyze data from the MWD RE J0317-853 to look for evidence of this process.

The couplings of the axion $a$ with mass $m_{a}$ to electromagnetism and electronic matter are described through the Lagrangian terms

$$
\mathcal{L}_{\text {int }} \supset-\frac{1}{4} g_{a \gamma \gamma} a F_{\mu \nu} \tilde{F}^{\mu \nu}+\frac{g_{a e e}}{2 m_{e}}\left(\partial_{\mu} a\right) \bar{e} \gamma^{\mu} \gamma_{5} e,
$$

with $F(\tilde{F})$ the (dual) quantum electrodynamics field strength, $e$ the electron field, and $m_{e}$ the electron mass. It is convenient to parametrize the coupling constants by $g_{a \gamma \gamma}=C_{a \gamma \gamma} \alpha_{\mathrm{EM}} /\left(2 \pi f_{a}\right)$ and $g_{a e e}=C_{a e e} m_{e} / f_{a}$, where the $C$ 's are dimensionless. Most laboratory and astrophysical searches for axions focus on the axion-photon coupling, with current constraints illustrated in Fig. 1. Low-mass constraints arise from the nonobservation of photons from super star clusters (SSCs) [29] (see also Ref. [30]) and SN1987A [31] and searches for spectral modulations with Fermi [32], H.E.S.S. [33], and Chandra [34] (but see Ref. [35]). The constraints from the solar axion search with the CAST experiment [36] and from horizontal branch (HB) star cooling [37] are equally strong and extend over the whole mass range in Fig. 1, which also shows the predicted coupling-mass relations in the DFSZ $[38,39]$ 


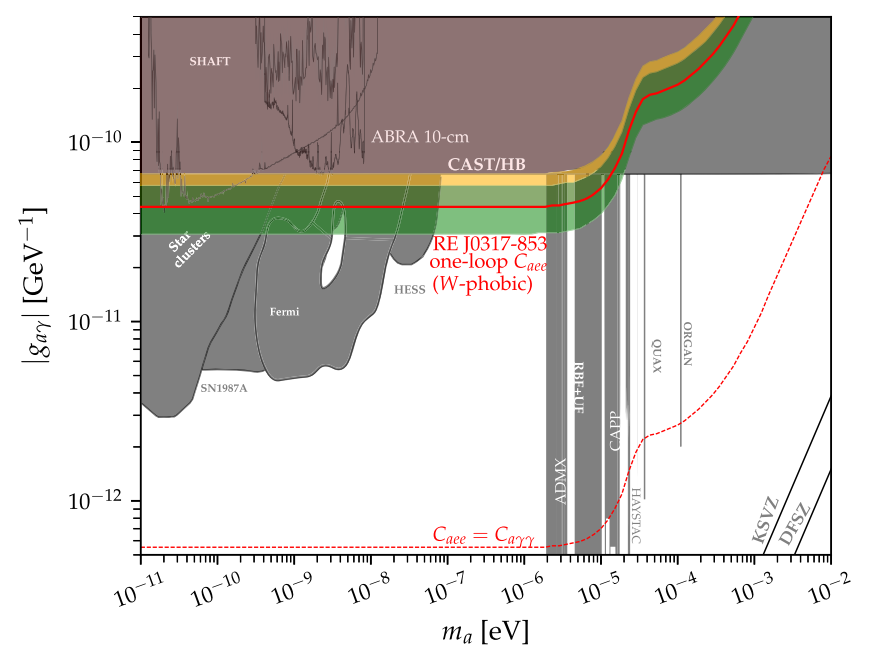

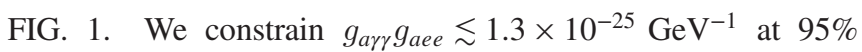
confidence for low $m_{a}$ from the nonobservation of $\mathrm{x}$ rays from the MWD RE J0317-853. We translate this result to constraints on $g_{a \gamma \gamma}$ assuming (i) a tree-level axion-electron coupling with $C_{a e e}=C_{a \gamma \gamma}$ and (ii) the loop-induced $C_{a e e} \approx 1.6 \times 10^{-4} C_{a \gamma \gamma}$ that represents a conservative $W$-phobic axion (the loop-induced $C_{a e e}$ is generically larger). The expected $68 \%$ (95\%) containment region for the power-constrained $95 \%$ upper limit is shaded in green (gold) for the $W$-phobic scenario. Previous constraints are shaded in gray [52].

and KSVZ [40,41] QCD axion models. The additional constraints shown in Fig. 1 require the axion to be dark matter [42-51] (see Ref. [52] for a summary).

As described in Ref. [18] axions may be produced within the cores of MWD stars through electron bremsstrahlung off of ions, using the $g_{a e e}$ coupling, and converted to x rays in the stellar magnetospheres with the $g_{a \gamma \gamma}$ term in Eq. (1). Reference [18] identified RE J0317-853 as being the most promising currently known MWD because of a combination of (i) the close distance $d=29.38 \pm 0.02 \mathrm{pc}$, as measured by Gaia [53], (ii) the large magnetic field $B_{\text {pole }} \sim 500 \mathrm{MG}$, and (iii) the high core temperature $T_{\text {core }} \sim 1.5 \mathrm{keV}$. The predicted axion-induced x-ray signal is expected to be roughly thermal at the core temperature, meaning that it should peak at a few $\mathrm{keV}$ where Chandra is the most sensitive currently operating x-ray telescope.

We observed the MWD RE J0317-853 on 2020-12-18 using the Chandra ACIS-I instrument with no grating for a total of $37.42 \mathrm{ks}$ (PI Safdi, Observation ID No. 22326). After data reduction-see the Supplemental Material [54] - we produce pixelated count maps in four energy bins from 1 to $9 \mathrm{keV}$ of width $2 \mathrm{keV}$ each. Each square pixel in right ascension (RA) and declination (dec) has physical length of $\sim 0^{\prime \prime} 492$ [note in the RA direction this is the width in RA $\times \cos (\mathrm{Dec})]$. In Fig. 2 we show the binned counts over $1-9 \mathrm{keV}$ in the vicinity of the MWD; note that in this region no pixel has more than one count. The figure is centered at the current location of the

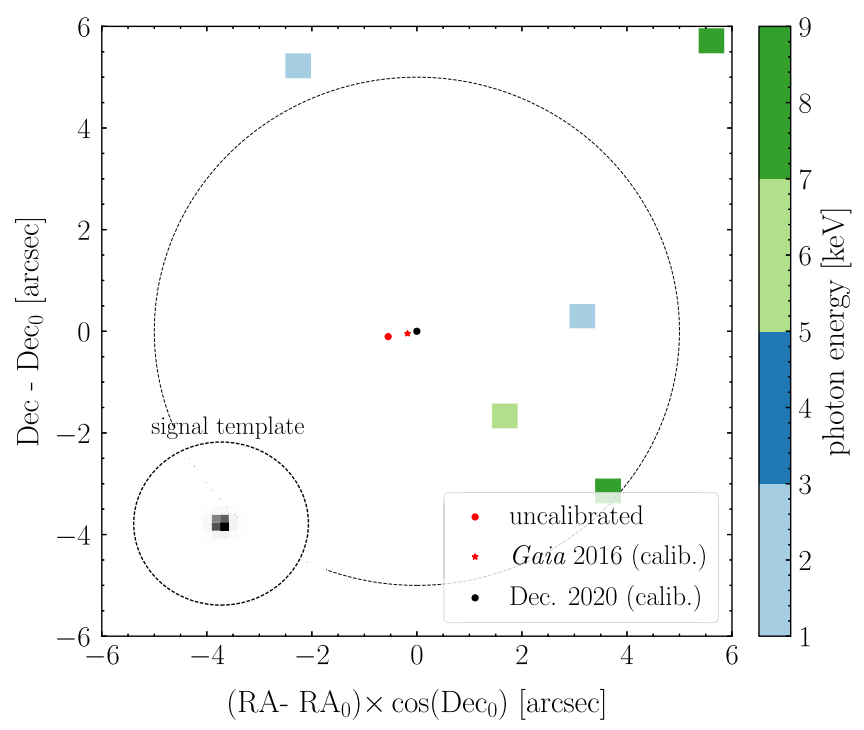

FIG. 2. The binned counts over $1-9 \mathrm{keV}$ from our $\sim 40 \mathrm{ks}$ Chandra observation of the MWD RE J0317-853. No counts are observed within the vicinity of the source, whose location is indicated along with intermediate locations at various stages in the astrometric calibration process (see text for details), and also no more than one count is observed in any pixel. The dashed circle indicates the extent of the ROI used in our analysis. Inset: the signal template in gray scale, for the first energy bin, over the analysis ROI.

MWD, labeled 'Dec. 2020 (calib.)': $\mathrm{RA}_{0} \approx 49^{\circ} 18^{\prime} 37^{\prime \prime} 77$, $\mathrm{DEC}_{0} \approx-85^{\circ} 32^{\prime} 25^{\prime \prime} .81$. Figure 2 also shows intermediate source locations determined during the astrometric calibration process (see the Supplemental Material [54]). The $68 \%$ energy containment radius at $1 \mathrm{keV}(9 \mathrm{keV})$ is approximately $0^{\prime \prime} 5\left(0^{\prime \prime} 6\right)$. The inset illustrates the expected template for emission associated with the MWD at $1 \mathrm{keV}$. No photon counts are observed near the MWD. The circle in Fig. 2 has radius $5^{\prime \prime}$ and is the extent of our region of interest (ROI); that is, we exclude pixels whose centers are beyond this radius in our analysis.

We analyze the pixelated data $\mathbf{d}=\left\{n_{i, j}\right\}$, with $n_{i, j}$ the number of counts in energy bin $i$ and pixel $j$, in the context of the axion model, which is discussed further shortly, using the joint Poisson likelihood

$$
p(\mathbf{d} \mid \mathcal{M}, \boldsymbol{\theta})=\prod_{i=1}^{4} \prod_{j=1}^{\mathrm{N}_{\mathrm{pix}}} \frac{\mu_{i, j}(\boldsymbol{\theta})^{n_{i, j}} e^{-\mu_{i, j}(\boldsymbol{\theta})}}{n_{i, j} !},
$$

with $\mathcal{M}$ denoting the joint signal and background model, with model parameters $\boldsymbol{\theta}=\left\{\mathbf{A}_{\mathrm{bkg}}, g_{\text {aee }} g_{\text {ary }}, m_{a}\right\}$, and $N_{\text {pix }}$ the number of spatial pixels. The model predicts $\mu_{i, j}(\boldsymbol{\theta})$ counts in energy and spatial pixel $i, j$. The background parameter vector $\mathbf{A}_{\mathrm{bkg}}$ consists of a single normalization parameter in each of the four energy bins that rescales the background counts' spatial template. For our background template, which we profile over, we use the exposure map, 
which is flat to less than $0.5 \%$ over our ROI. The signal model has the two parameters $\left\{g_{a e e} g_{a \gamma \gamma}, m_{a}\right\}$, which predict the counts in each of the four energy bins. The signal template is centered on the MWD and accounts for the point spread function (PSF), as illustrated in the inset of Fig. 2.

At a fixed $m_{a}$ we construct the profile likelihood for $g_{\text {ayy }} g_{\text {aee }}$ by maximizing the $\log$-likelihood over $\mathbf{A}_{\text {bkg }}$ at each $g_{a \gamma \gamma} g_{a e e}$. Our 95\% upper limit on $g_{a \gamma \gamma} g_{a e e}$ is constructed directly by Monte Carlo simulations of the signal and null hypotheses instead of relying on Wilks' theorem, since we are in the low-counts limit (see, e.g., [60] for details). A priori we decided to power constrain [61] our limits to account for the possibility of under fluctuations, though this was not necessary in practice.

We also analyze the data using the Poisson likelihood in the individual energy bins to extract the spectrum $d F / d E$, which is illustrated in Fig. 3. In that figure we overlay the axion model prediction, which we now detail. For production via axion bremsstrahlung from electron-ion scattering $[16,62]$, we broadly follow the formalism developed in Ref. [18], though we make improvements thanks to updated WD models and luminosity data from Gaia. Firstly, we improve our modeling of the density profile and composition of RE J0317-853 using MESA [63] version 12778. We simulate a WD of RE J0317-853's mass from stellar birth until it has cooled below RE J0317-853's observed luminosity. These simulations account for core electrostatic effects including ionic correlations and crystallization in the core that modify the profiles from that of a fully degenerate ideal electron gas, which were neglected in

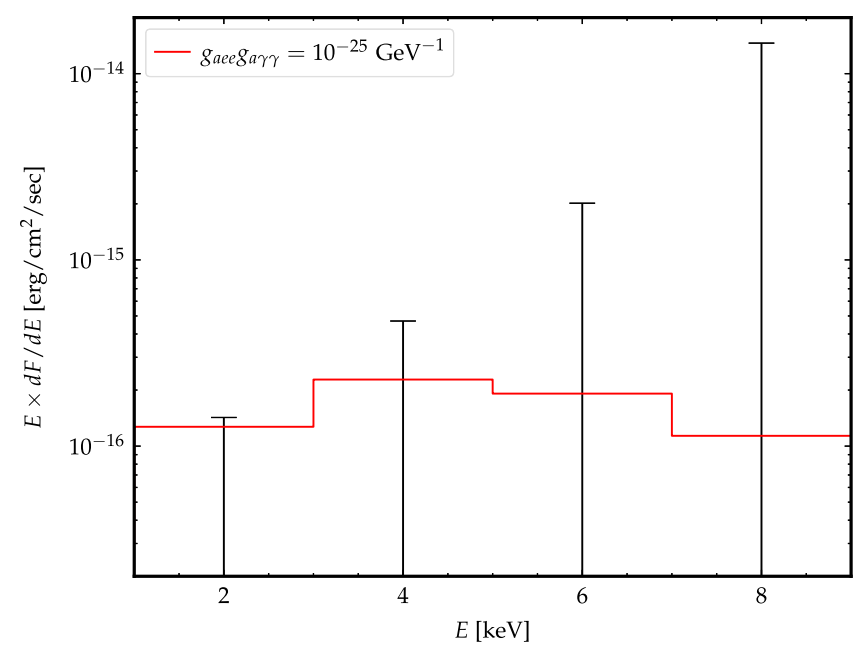

FIG. 3. The energy spectrum found from our analysis of the Chandra data from the MWD RE J0317-853. In each of the four energy bins the best-fit fluxes are consistent with zero (the $68 \%$ containment intervals are shown). We also illustrate the predicted axion-induced signal that would be seen from an axion with the indicated couplings and $m_{a} \ll 10^{-5} \mathrm{eV}$.
Ref. [18]. We find RE J0317-853 has a predominantly oxygen-neon core because it completed carbon burning while ascending the asymptotic giant branch, typical for a WD of its mass undergoing single-star evolution. We take as our fiducial profiles those density and composition profiles from the model for which the luminosity matches the observed luminosity of RE J0317-853 (see Sec. IV of the Supplemental Material [54] for further details).

The second improvement we make is in estimating the core temperature of RE J0317-853. Reference [18] estimated the core temperature from an empirical core temperature-luminosity relation using an assumed luminosity from Ref. [64]. Reference [64] used Hubble parallax and photometric data along with WD cooling sequences to estimate the luminosity of RE J0317-853. Here, we estimate the core temperature from WD cooling sequences [65] which predict Gaia DR2 band magnitudes. These cooling sequences are improved over those of Ref. [64] because they better account for ionic correlation effects than previous sequences, and our use of Gaia data rather than Hubble represents an improvement because of smaller uncertainties on the magnitudes, partly due to improved parallax measurements. In particular, we fit the models in Ref. [65] over cooling age and mass to the measured RE J0317-853 Gaia DR2 data [66]. Although previous measurements indicated a mass for RE J0317-853 of $\gtrsim 1.26 M_{\odot}$, we find that the $1.22 M_{\odot}$ model provides the best fit to the data. In the context of that model, we find that the Gaia data prefers a core temperature $T_{c}=1.388 \pm 0.005 \mathrm{keV}$. Therefore we use this model and to be conservative assume a core temperature at the lower $1 \sigma$ allowed value, $T_{c}=1.383 \mathrm{keV}$, since the emissivity increases with increasing $T_{c}$.

Axion emission from the stellar interior primarily results from the bremsstrahlung scattering $e+(A, Z) \rightarrow$ $e+(A, Z)+a$ where an electron is incident on a nucleus with atomic number $Z$ and mass number $A$. The electrons in a WD core are strongly degenerate with a temperature $T \ll p_{F}$ that is much smaller than the Fermi momentum $p_{F}$. In this regime, the axion emissivity spectrum is thermal and given by $[16,18,62,67]$

$$
\frac{d \varepsilon_{a}}{d \omega}=\frac{\alpha_{\mathrm{EM}}^{2} g_{a e e}^{2}}{4 \pi^{3} m_{e}^{2}} \frac{\omega^{3}}{e^{\omega / T}-1} \sum_{s} \frac{Z_{s}^{2} \rho_{s} F_{s}}{A_{s} u},
$$

which includes a sum over the species $s$ of nuclei that are present in the plasma; $Z_{s}$ is the atomic number, $A_{s}$ is the mass number, $\rho_{s}$ is the mass density, and $u \simeq 931.5 \mathrm{MeV}$ is the atomic mass unit. The species-dependent, order-one dimensionless factor $F_{s}$ accounts for medium effects, including screening of the electric field and interference between different scattering sites. For a strongly coupled plasma [68] we use the empirical fitting functions provided by Ref. [67]. Whereas Ref. [67] assumes a onecomponent plasma, we follow Ref. [69] and generalize to a 
multicomponent plasma in Eq. (3) by summing over the constituent ionic species; this approach assumes that possible interference effects can be neglected. Note that the axion luminosity is given by the integral of the emissivity over the WD core.

Our fiducial WD model leads to the predicted axion luminosity $L_{a} \approx 8 \times 10^{-4} L_{\odot}\left(g_{a e e} / 10^{-13}\right)^{2}$. Accounting for modeling uncertainties on RE J0317-853 we estimate the limit on $g_{\text {ary }}$ may be $\sim 10 \%$ stronger, as illustrated in the Supplemental Material, Fig. S4 [54]. Axions may also be produced by the $g_{a \gamma \gamma}$ coupling from electro-Primakoff production, which we compute in the Supplemental Material, though as we show in the Supplemental Material Figs. S2 and S3 [54], this process is subdominant compared with bremsstrahlung for RE J0317-853.

The axions then undergo conversion to $\mathrm{x}$ rays in the MWD magnetic fields. The conversion probability $p_{a \rightarrow \gamma}$ may be calculated numerically for arbitrary magnetic field configurations and axion masses $m_{a}$ by solving the axion-photon mixing equations in the presence of $g_{\text {ary }}$, though it is important to incorporate the Euler-Heisenberg Lagrangian term which modifies the propagation of photons in strong magnetic fields and suppresses the mixing [20]. The magnetic field of the MWD is found to vary over the rotation period between $200 \mathrm{MG}$ and $800 \mathrm{MG}$ [70]; we follow Ref. [18] and assume a dipole field of strength 200 $\mathrm{MG}$, to be conservative. Note that at low axion masses and high $B$-field values the dependence of the conversion probability on the magnetic field is mild: $p_{a \rightarrow \gamma} \propto B^{2 / 5}$ [18]. Using the offset dipole model from Ref. [70] increases the conversion probabilities by up to $\sim 50 \%$ [18] at low masses, which may increase the limit by $\sim 10 \%$ relative to our fiducial case. Numerically the conversion probabilities

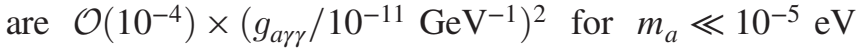
and drop off for higher masses. The distance is fixed at the central value measured by Gaia $d=29.38$ pc [53] because the distance uncertainty only leads to a $\sim 0.1 \%$ uncertainty on the flux. In Fig. 3 we illustrate the energybinned spectrum prediction from axion-induced emission from the MWD for $m_{a} \ll 10^{-5} \mathrm{eV}$ and $g_{a e e} g_{a \gamma \gamma}=$ $10^{-25} \mathrm{GeV}^{-1}$. Note we neglect conversion in the Galactic magnetic fields, which we estimate to be subdominant to that in the WD magnetosphere.

We find no evidence for the axion model, with the bestfit coupling combination being zero for all masses. We thus set $95 \%$ one-sided upper limits on the coupling combination $g_{a e e} g_{a \gamma y}$ at fixed axion masses $m_{a}$ using the profile likelihood procedure. For low masses $m_{a} \ll 10^{-5} \mathrm{eV}$ the limit is $g_{\text {aee }} g_{\text {ary }} \lesssim 1.3 \times 10^{-25} \mathrm{GeV}^{-1}$. This limit is around 3 orders of magnitude stronger than that set by the CAST experiment on this coupling combination [36]. Our limit also severely constrains the low-mass axion explanation of stellar cooling anomalies [17], which prefer $g_{\text {ayr }} g_{a e e} \sim$ $2 \times 10^{-24} \mathrm{GeV}^{-1}$ as illustrated in Fig. 4, where we show

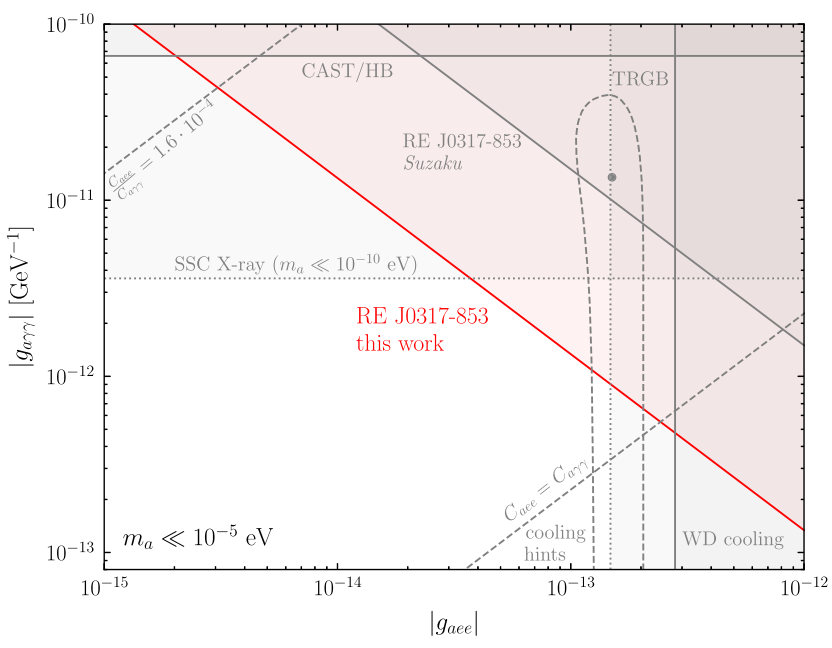

FIG. 4. The $95 \%$ one-sided limit on the axion-photon and axion-

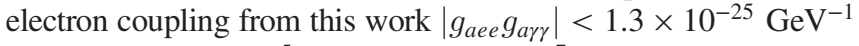
assuming $m_{a} \ll 10^{-5} \mathrm{eV}$. For $m_{a} \gtrsim 10^{-7} \mathrm{eV}$ the leading constraint on $g_{a \gamma \gamma}$ is from the CAST experiment [36] and HB star cooling [37], while for $m_{a} \lesssim 10^{-10} \mathrm{eV}$ it is from x-ray observations of SSCs [29]. The leading limits on $g_{\text {aee }}$ are from WD cooling [71] and TRGB observations [72,73], while the $68 \%$ containment region for explaining stellar cooling anomalies [17], along with the best-fit coupling, is also indicated and in tension with our null results. In the regime that is excluded by WD cooling, our analysis should be modified to account for stellar energy loss into axions. Dashed diagonal lines show the relations $C_{a e e} / C_{a \gamma \gamma}=1$ and also $C_{\text {aee }} / C_{\text {ary }}=1.6 \times 10^{-4}$, the latter corresponding to the $W$-phobic loop-induced axion-electron coupling.

our low-mass limit in the $g_{a \gamma \gamma}-g_{a e e}$ plane, along with current constraints.

It is instructive to translate our limit to one on $g_{a y \gamma}$ alone by assuming a relation between the dimensionless coupling constants $C_{a e e}$ and $C_{a \gamma \gamma}$. Note that in the DFSZ QCD axion model there is a tree-level coupling between the axion and electron, such that $C_{a e e} \sim C_{a \gamma \gamma}$, while in the KSVZ model no ordinary matter is charged under the Peccei-Quinn symmetry and so $C_{a e e}=0$ at tree level, though it is generated at one loop [74]. The loop-induced value of $C_{a \gamma \gamma}$ depends on the relative coupling of the axion to $\mathrm{SU}(2)_{L}$ versus hypercharge $\mathrm{U}(1)_{Y}$. If the axion couples only to $\mathrm{SU}(2)_{L}\left[\mathrm{U}(1)_{Y}\right]$ then we expect, at one loop, $C_{a e e} \sim 4.8 \times 10^{-4} C_{a \gamma \gamma}\left(C_{a e e} \sim 1.6 \times 10^{-4} C_{a \gamma \gamma}\right)$ for $f_{a} \approx$ $10^{9} \mathrm{GeV}^{-1}$ (see Refs. [18,74,75] and the Supplemental Material [54]). To be conservative we assume in Fig. 1 the $W$-phobic axion scenario, where the axion only couples to $\mathrm{U}(1)_{Y}$ (but see the Supplemental Material, Fig. S2 [54]). We also show the limit on $g_{a \gamma \gamma}$ for axion models with $C_{a e e}=C_{a \gamma \gamma}$, which is nearly 2 orders of magnitude stronger than the loop-induced limit. Note, however, that assuming $C_{a e e}=C_{a \gamma \gamma}$ allows constraints on $g_{a e e}$ from WD cooling [71] and tip of the red giant branch (TRGB) 
observations $[72,73]$ to be recast as constraints on $g_{a \gamma \gamma}$ at the level of few $\times 10^{-13} \mathrm{GeV}^{-1}$, which are not shown in Fig. 1. Couplings $C_{a e e} \sim C_{a \gamma \gamma}$ may arise naturally in models with ultraviolet operators of the form $a \bar{e} \gamma_{5} e$.

Our results have strong implications for a number of astrophysical anomalies and planned laboratory experiments. For example, the WD cooling anomaly prefers $g_{\text {aee }} \sim$ $1.6 \times 10^{-13}$ [17]. In order for a low-mass axion to explain this result and be compatible with our upper limit, one would need $C_{a \gamma \gamma} \lesssim 2.2 C_{a e e}\left(g_{a \gamma \gamma} \lesssim 8.1 \times 10^{-13} \mathrm{GeV}^{-1}\right)$, which would not be able to also explain the axion-photon coupling $g_{\text {ary }} \sim 10^{-11} \mathrm{GeV}^{-1}$ suggested by the global fit to stellar cooling data [17] (see Fig. 4) or the $\mathrm{TeV}$ transparency anomalies, which prefer $g_{\text {ary }} \gtrsim 2 \times 10^{-11} \mathrm{GeV}^{-1}$ for $m_{a} \ll$ $10^{-8} \mathrm{eV}$ [76]. Anomalous x-ray emission from nearby isolated Magnificent Seven NSs may be interpreted as low-mass $\left(m_{a} \ll 10^{-5} \mathrm{eV}\right)$ axion production from nucleon bremsstrahlung in the NS cores and conversion to $\mathrm{x}$ rays in the NS magnetospheres [27,77]. The required coupling combination to explain the $\mathrm{x}$-ray excesses is $g_{a \gamma \gamma} g_{a N N} \gtrsim 10^{-21} \mathrm{GeV}^{-1}$, with $g_{a N N}=C_{a N N} m_{N} / f_{a}$ the axion-nucleon coupling, with $m_{N}$ the nucleon mass and $C_{a N N}$ the dimensionless coupling. The nonobservation of $\mathrm{x}$ rays in this work from the MWD implies that if axions explain the Magnificent Seven excess they must be electrophobic, with $C_{a e e} \lesssim 4 C_{a N N}$. Lastly, we note that our results are especially relevant for the upcoming ALPS II lightshining-through-walls experiment [78]. The last stage of the experiment will have sensitivity to $g_{\text {ary }} \gtrsim 2 \times 10^{-11} \mathrm{GeV}^{-1}$ for $m_{a} \lesssim 10^{-4} \mathrm{eV}$, meaning that much of the axion parameter space to be probed is constrained by the current analysis (see the Supplemental Material, Fig. S2 [54]).

As is evident in, e.g., Fig. 2 with $\sim 40$ ks of Chandra data we are able to perform a nearly zero-background search; an additional order of magnitude in exposure time would allow us to improve the sensitivity to $g_{a \gamma \gamma}$ by a factor of $\sim 1.5$. The proposed Lynx X-ray Observatory [79] aims to improve the point source sensitivity by roughly 2 orders of magnitude compared with Chandra. A $\sim 400 \mathrm{ks}$ observation with Lynx or a similar future telescope of RE J0317-853 (see the Supplemental Material, Fig. S1 [54]) may be sensitive to axions with $g_{a \gamma \gamma} \sim 10^{-13} \mathrm{GeV}^{-1}$ for $C_{a e e} \sim C_{a \gamma \gamma}$, which may probe photophilic QCD axion models [5-8] in addition to vast regions of uncharted parameter space for the hypothetical axiverse.

We thank Josh Foster and Anson Hook for useful conversations. A. J. L. was supported in part by the National Science Foundation under Grant No. 2114024. C. D. and B. R. S. were supported in part by the DOE Early Career Grant No. DESC0019225. This research used resources from the National Energy Research Scientific Computing Center (NERSC) and the Lawrencium computational cluster provided by the IT Division at the Lawrence
Berkeley National Laboratory, supported by the Director, Office of Science, and Office of Basic Energy Sciences, of the U.S. Department of Energy under Contract No. DEAC02-05CH11231. Support for this work was provided by the National Aeronautics and Space Administration through Chandra Grant No. GO0-21013X issued by the Chandra X-ray Center (CXC), which is operated by the Smithsonian Astrophysical Observatory for and on behalf of the National Aeronautics Space Administration under Contract No. NAS8-03060. The scientific results reported in this article are based to a significant degree on observations made by the Chandra X-ray Observatory. This research has made use of software provided by the CXC in the application package CIAO.

[1] R. D. Peccei and H. R. Quinn, CP Conservation in the Presence of Instantons, Phys. Rev. Lett. 38, 1440 (1977).

[2] R. D. Peccei and H. R. Quinn, Constraints Imposed by CP conservation in the presence of instantons, Phys. Rev. D 16, 1791 (1977).

[3] S. Weinberg, A New Light Boson?, Phys. Rev. Lett. 40, 223 (1978).

[4] F. Wilczek, Problem of Strong p and t Invariance in the Presence of Instantons, Phys. Rev. Lett. 40, 279 (1978).

[5] M. Farina, D. Pappadopulo, F. Rompineve, and A. Tesi, The photo-philic QCD axion, J. High Energy Phys. 01 (2017) 095.

[6] L. Di Luzio, F. Mescia, and E. Nardi, Window for preferred axion models, Phys. Rev. D 96, 075003 (2017).

[7] L. Darmé, L. Di Luzio, M. Giannotti, and E. Nardi, Selective enhancement of the QCD axion couplings, Phys. Rev. D 103, 015034 (2021).

[8] A. V. Sokolov and A. Ringwald, Photophilic hadronic axion from heavy magnetic monopoles, J. High Energy Phys. 06 (2021) 123.

[9] P. Svrcek and E. Witten, Axions in string theory, J. High Energy Phys. 06 (2006) 051.

[10] A. Arvanitaki, S. Dimopoulos, S. Dubovsky, N. Kaloper, and J. March-Russell, String axiverse, Phys. Rev. D 81, 123530 (2010).

[11] B. S. Acharya, K. Bobkov, and P. Kumar, An M theory solution to the strong $\mathrm{CP}$ problem and constraints on the axiverse, J. High Energy Phys. 11 (2010) 105.

[12] A. Ringwald, Searching for axions and ALPs from string theory, J. Phys. Conf. Ser. 485, 012013 (2014).

[13] M. J. Stott, D. J. E. Marsh, C. Pongkitivanichkul, L. C. Price, and B. S. Acharya, Spectrum of the axion dark sector, Phys. Rev. D 96, 083510 (2017).

[14] J. Halverson, C. Long, B. Nelson, and G. Salinas, Towards string theory expectations for photon couplings to axionlike particles, Phys. Rev. D 100, 106010 (2019).

[15] G. G. Raffelt, Axion constraints from white dwarf cooling times, Phys. Lett. 166B, 402 (1986).

[16] G. G. Raffelt, Astrophysical methods to constrain axions and other novel particle phenomena, Phys. Rep. 198, 1 (1990). 
[17] M. Giannotti, I. G. Irastorza, J. Redondo, A. Ringwald, and K. Saikawa, Stellar recipes for axion hunters, J. Cosmol. Astropart. Phys. 10 (2017) 010.

[18] C. Dessert, A. J. Long, and B. R. Safdi, X-ray Signatures of Axion Conversion in Magnetic White Dwarf Stars, Phys. Rev. Lett. 123, 061104 (2019).

[19] D. E. Morris, Axion mass limits from pulsar X-rays, Phys. Rev. D 34, 843 (1986).

[20] G. Raffelt and L. Stodolsky, Mixing of the photon with low mass particles, Phys. Rev. D 37, 1237 (1988).

[21] D. Lai and J. Heyl, Probing axions with radiation from magnetic stars, Phys. Rev. D 74, 123003 (2006).

[22] D. Chelouche, R. Rabadan, S. Pavlov, and F. Castejon, Spectral signatures of photon-particle oscillations from celestial objects, Astrophys. J. Suppl. Ser. 180, 1 (2009).

[23] R. Jimenez, C. Pena-Garay, and L. Verde, Is it possible to explore Peccei-Quinn axions from frequency-dependence radiation dimming?, Phys. Lett. B 703, 232 (2011).

[24] R. Perna, W. C. G. Ho, L. Verde, M. van Adelsberg, and R. Jimenez, Signatures of photon-axion conversion in the thermal spectra and polarization of neutron stars, Astrophys. J. 748, 116 (2012).

[25] J.-F. Fortin and K. Sinha, Constraining axion-like-particles with hard X-ray emission from magnetars, J. High Energy Phys. 06 (2018) 048.

[26] J.-F. Fortin and K. Sinha, X-ray polarization signals from magnetars with axion-like-particles, J. High Energy Phys. 01 (2019) 163.

[27] M. Buschmann, R. T. Co, C. Dessert, and B. R. Safdi, Axion Emission Can Explain a New Hard X-Ray Excess from Nearby Isolated Neutron Stars, Phys. Rev. Lett. 126, 021102 (2021).

[28] J.-F. Fortin, H.-K. Guo, S. P. Harris, E. Sheridan, and K. Sinha, Magnetars and axion-like particles: Probes with the gard X-ray spectrum, J. Cosmol. Astropart. Phys. 06 (2021) 036.

[29] C. Dessert, J. W. Foster, and B. R. Safdi, X-ray Searches for Axions from Super Star Clusters, Phys. Rev. Lett. 125, 261102 (2020).

[30] M. Xiao, K. M. Perez, M. Giannotti, O. Straniero, A. Mirizzi, B. W. Grefenstette, B. M. Roach, and M. Nynka, Constraints on Axionlike Particles from a Hard X-Ray Observation of Betelgeuse, Phys. Rev. Lett. 126, 031101 (2021).

[31] A. Payez, C. Evoli, T. Fischer, M. Giannotti, A. Mirizzi, and A. Ringwald, Revisiting the SN1987A gamma-ray limit on ultralight axion-like particles, J. Cosmol. Astropart. Phys. 02 (2015) 006.

[32] M. Ajello et al. (Fermi-LAT Collaboration), Search for Spectral Irregularities due to Photon-Axionlike-Particle Oscillations with the Fermi Large Area Telescope, Phys. Rev. Lett. 116, 161101 (2016).

[33] A. Abramowski et al. (H.E.S.S. Collaboration), Constraints on axionlike particles with H.E.S.S. from the irregularity of the PKS 2155-304 energy spectrum, Phys. Rev. D 88, 102003 (2013).

[34] C. S. Reynolds, M. C. David Marsh, H. R. Russell, A. C. Fabian, R. Smith, F. Tombesi, and S. Veilleux, Astrophysical limits on very light axion-like particles from Chandra grating spectroscopy of NGC 1275, Astrophys. J. 890, 59 (2020).

[35] M. Libanov and S. Troitsky, On the impact of magnetic-field models in galaxy clusters on constraints on axion-like particles from the lack of irregularities in high-energy spectra of astrophysical sources, Phys. Lett. B 802, 135252 (2020).

[36] V. Anastassopoulos et al. (CAST Collaboration), New CAST limit on the axion-photon interaction, Nat. Phys. 13, 584 (2017).

[37] A. Ayala, I. Domínguez, M. Giannotti, A. Mirizzi, and O. Straniero, Revisiting the Bound on Axion-Photon Coupling from Globular Clusters, Phys. Rev. Lett. 113, 191302 (2014).

[38] M. Dine, W. Fischler, and M. Srednicki, A simple solution to the strong $\mathrm{CP}$ problem with a harmless axion, Phys. Lett. 104B, 199 (1981).

[39] A. R. Zhitnitsky, On possible suppression of the axion hadron interactions. (In Russian), Sov. J. Nucl. Phys. 31, 260 (1980).

[40] J. E. Kim, Weak Interaction Singlet and Strong CP Invariance, Phys. Rev. Lett. 43, 103 (1979).

[41] M. A. Shifman, A. I. Vainshtein, and V. I. Zakharov, Can confinement ensure natural $\mathrm{CP}$ invariance of strong interactions? Nucl. Phys. B166, 493 (1980).

[42] A. V. Gramolin, D. Aybas, D. Johnson, J. Adam, and A. O. Sushkov, Search for axion-like dark matter with ferromagnets, Nat. Phys. 17, 79 (2021).

[43] J. L. Ouellet, C. P. Salemi, J. W. Foster, R. Henning, Z. Bogorad, J. M. Conrad, J. A. Formaggio, Y. Kahn, J. Minervini, A. Radovinsky, N. L. Rodd, B. R. Safdi, J. Thaler, D. Winklehner, and L. Winslow, First Results from ABRACADABRA-10 cm: A Search for Sub- $\mu \mathrm{eV}$ Axion Dark Matter, Phys. Rev. Lett. 122, 121802 (2019).

[44] C. P. Salemi et al., The Search for low-Mass Axion Dark Matter with ABRACADABRA-10 cm, Phys. Rev. Lett. 127, 081801 (2021).

[45] N. Du, N. Force, R. Khatiwada, E. Lentz, R. Ottens et al. (ADMX Collaboration), A Search for Invisible Axion Dark Matter with the Axion Dark Matter Experiment, Phys. Rev. Lett. 120, 151301 (2018).

[46] T. Braine, R. Cervantes, N. Crisosto, N. Du, S. Kimes et al. (ADMX Collaboration), Extended Search for the Invisible Axion with the Axion Dark Matter Experiment, Phys. Rev. Lett. 124, 101303 (2020).

[47] L. Zhong, S. AlKenany, K. M. Backes, B. M. Brubaker, S. B. Cahn et al. (HAYSTAC Collaboration), Results from phase 1 of the HAYSTAC microwave cavity axion experiment, Phys. Rev. D 97, 092001 (2018).

[48] K. M. Backes et al. (HAYSTAC Collaboration), A quantumenhanced search for dark matter axions, Nature (London) 590, 238 (2021).

[49] J. Jeong, S. Youn, S. Bae, J. Kim, T. Seong, J. E. Kim, and Y. K. Semertzidis, Search for Invisible Axion Dark Matter with a Multiple-Cell Haloscope, Phys. Rev. Lett. 125, 221302 (2020).

[50] D. Alesini et al., Search for invisible axion dark matter of mass $\mathrm{m}_{a}=43 \mu \mathrm{eV}$ with the QUAX-ar experiment, Phys. Rev. D 103, 102004 (2021). 
[51] B. T. McAllister, G. Flower, E. N. Ivanov, M. Goryachev, J. Bourhill, and M.E. Tobar, The ORGAN experiment: An axion haloscope above $15 \mathrm{GHz}$, Phys. Dark Universe 18, 67 (2017).

[52] Ciaran O'HARE, cajohare/axionlimits: Axionlimits (2020), 10.5281/zenodo.3932429.

[53] A. G. A. Brown, A. Vallenari, T. Prusti, J. H. J. de Bruijne, C. Babusiaux, and M. Biermann (Gaia Collaboration), Gaia early data release 3: Summary of the contents and survey properties, Astron. Astrophys. 650, C3 (2021).

[54] See Supplemental Material at http://link.aps.org/ supplemental/10.1103/PhysRevLett.128.071102 for supplemental figures, our data reduction procedure, the renormalization group evolution of the axion-electron coupling, our modeling of the MWD RE J0317-853, and our calculation of the electro-Primakoff axion production rate, which includes Ref. [55-59].

[55] A. Fruscione et al., CIAO: Chandra's data analysis system, in Society of Photo-Optical Instrumentation Engineers (SPIE) Conference Series, edited by D. R. Silva and R. E. Doxsey, Society of Photo-Optical Instrumentation Engineers (SPIE) Conference Series Vol. 6270 (2006), p. 62701V, 10.1117/12.671760.

[56] M. C. Weisskopf, T. L. Aldcroft, M. Bautz, R. A. Cameron, D. Dewey, J. J. Drake, C. E. Grant, H. L. Marshall, and S. S. Murray, An overview of the performance of the Chandra X-Ray observatory, Exp. Astron. 16, 1 (2003).

[57] N. J. Secrest, R. P. Dudik, B. N. Dorland, N. Zacharias, V. Makarov, A. Fey, J. Frouard, and C. Finch, Identification of 1.4 Million Active Galactic Nuclei in the Mid-Infrared using WISE Data, Astrophys. J. Suppl. Ser. 221, 12 (2015).

[58] M. Bauer, M. Neubert, S. Renner, M. Schnubel, and A. Thamm, The low-energy effective theory of axions and ALPs, J. High Energy Phys. 04 (2021) 063.

[59] A. Bédard, P. Bergeron, P. Brassard, and G. Fontaine, On the spectral evolution of hot white dwarf stars. I. A detailed model atmosphere analysis of hot white dwarfs from SDSS DR12, Astrophys. J. 901, 93 (2020).

[60] G. Cowan, K. Cranmer, E. Gross, and O. Vitells, Asymptotic formulae for likelihood-based tests of new physics, Eur. Phys. J. C 71, 1554 (2011); 73, 2501(E) (2013).

[61] G. Cowan, K. Cranmer, E. Gross, and O. Vitells, Powerconstrained limits, arXiv:1105.3166.

[62] M. Nakagawa, Y. Kohyama, and N. Itoh, Axion bremsstrahlung in dense stars, Astrophys. J. 322, 291 (1987).

[63] B. Paxton, L. Bildsten, A. Dotter, F. Herwig, P. Lesaffre, and F. Timmes, Modules for experiments in stellar astrophysics (mesa), Astrophys. J. Suppl. Ser. 192, 3 (2011).

[64] B. Kulebi, S. Jordan, E. Nelan, U. Bastian, and M. Altmann, Constraints on the origin of the massive, hot, and rapidly rotating magnetic white dwarf RE J 0317-853 from an HST parallax measurement, Astron. Astrophys. 524, A36 (2010).
[65] M. E. Camisassa, L. G. Althaus, A. H. Córsico, F. C. De Gerónimo, M. M. Miller Bertolami, M. L. Novarino, R. D. Rohrmann, F. C. Wachlin, and E. García-Berro, The evolution of ultra-massive white dwarfs, Astron. Astrophys. 625, A87 (2019).

[66] A. G. A. Brown, A. Vallenari, T. Prusti, J. H. J. de Bruijne, C. Babusiaux, C. A. L. Bailer-Jones, M. Biermann, D. W. Evans, L. Eyer et al., Gaia data release 2, Astron. Astrophys. 616, A1 (2018).

[67] M. Nakagawa, T. Adachi, Y. Kohyama, and N. Itoh, Axion bremsstrahlung in dense stars. II-Phonon contributions, Astrophys. J. 326, 241 (1988).

[68] S. Ichimaru, Strongly coupled plasmas: High-density classical plasmas and degenerate electron liquids, Rev. Mod. Phys. 54, 1017 (1982).

[69] N. Itoh, H. Hayashi, A. Nishikawa, and Y. Kohyama, Neutrino energy loss in stellar interiors. VII. Pair, photo-, plasma, bremsstrahlung, and recombination neutrino processes, Astrophys. J. Suppl. Ser. 102, 411 (1996).

[70] M. R. Burleigh, S. Jordan, and W. Schweizer, Phaseresolved far-ultraviolet hst spectroscopy of the peculiar magnetic white dwarf re j0317-853, Astrophys. J. Lett. 510, L37 (1999).

[71] M. M. Miller Bertolami, B. E. Melendez, L. G. Althaus, and J. Isern, Revisiting the axion bounds from the Galactic white dwarf luminosity function, J. Cosmol. Astropart. Phys. 10 (2014) 069.

[72] F. Capozzi and G. Raffelt, Axion and neutrino bounds improved with new calibrations of the tip of the red-giant branch using geometric distance determinations, Phys. Rev. D 102, 083007 (2020).

[73] O. Straniero, C. Pallanca, E. Dalessandro, I. Dominguez, F. R. Ferraro, M. Giannotti, A. Mirizzi, and L. Piersanti, The RGB tip of galactic globular clusters and the revision of the axion-electron coupling bound, Astron. Astrophys. 644, A166 (2020).

[74] M. Srednicki, Axion couplings to matter. 1. CP conserving parts, Nucl. Phys. B260, 689 (1985).

[75] S. Chang and K. Choi, Hadronic axion window and the big bang nucleosynthesis, Phys. Lett. B 316, 51 (1993).

[76] M. Meyer, D. Horns, and M. Raue, First lower limits on the photon-axion-like particle coupling from very high energy gamma-ray observations, Phys. Rev. D 87, 035027 (2013).

[77] C. Dessert, J. W. Foster, and B. R. Safdi, Hard X-ray excess from the magnificent seven neutron stars, Astrophys. J. 904, 42 (2020).

[78] R. Bähre et al., Any light particle search II-Technical design report, J. Instrum. 8, T09001 (2013).

[79] The Lynx Team, The Lynx mission concept study interim report, arXiv:1809.09642. 